\title{
A new method for quantitative evaluation of target volume variations in radiotherapy planning
}

\author{
F. Gaya ${ }^{1,4}$, B. Rodriguez-Vila ${ }^{1,2}$, F. del Pozo ${ }^{1,2}$, F. Garcia-Vicente ${ }^{3}$ and E.J. Gomez ${ }^{1,2}$ \\ ${ }^{1}$ Bioengineering and Telemedicine Group, Polytechnical University of Madrid, Madrid, Spain \\ ${ }^{2}$ Networking Research on Bioengineering, Biomaterials and Nanomedicine (CIBER-BBN), Madrid, Spain \\ ${ }^{3}$ Medical Physics, Radiotherapy Department, University Hospital of La Princesa, Madrid, Spain \\ ${ }^{4}$ Section of Biostatistics, La Paz University Hospital, Madrid, Spain
}

\begin{abstract}
A new method for three-dimensional quantitative evaluation of target volume delineation is presented. It is composed by a new $3 \mathrm{D}$ reconstruction method called Origami, based on the combination of two bidimensional analysis of the volume of each organ instead of one 3D analysis, and a 3D description of the error distribution in the space. The Origami method avoid external errors introduced by a 3D rendering and has shown to work correctly in both convex and convex-concave volumes, accurately fitting the contours of the planning study. Its performance has been evaluated comparing it with the Convex Hull algorithm using Delaunay triangulation, resulting in a much more adjusted evaluation. Moreover, Origami computes thousands of control points in less than one second in a common PC.
\end{abstract}

Keywords-Quantitative evaluation, target volume delineation, radiotherapy planning.

\section{INTRODUCTION}

Three-dimensional conformal radiotherapy (3D-CRT) tries to deliver a high dose of radiation to the tumor, sparing the surrounding normal tissue to a maximum extent at the same time [1]. Gross Tumor Volume (GTV) is defined in a first stage in order to facilitate the three-dimensional treatment process, and manual outlining of the contours of the GTV in each slice of the planning study is one of the most time expensive tasks of the process. Clinical Target Volume (CTV) and Planning Target Volume (PTV) are derived from GTV, so its delineation has a great influence in the whole planning process [2]. The reproducibility and accuracy of delineation are not very well known, and it has been shown that the shape and size of the GTV have significant inter-specialist, and also intra-specialist, variations.

The 3D nature of 3D-CRT requires a full 3D description of the variability of the target volume delineation. The work of Remeijer et al. [3] approaches the problem through a general methodology for the analysis of the variations in the delineation, while the article of Pevsner et al. [4] introduces some changes in this methodology in order to evaluate the performance of a non-rigid registration algorithm. In these studies of reference, the 3D surface is rendered from the contours by means of some kind of tessellation. These geometrical methods have some problems to work correctly in convex-concave structures. However the volumes of the organs in the human body, such as bladder or prostate including the seminal vesicles, have some concavities that can introduce a relevant error in the evaluation.

Non-rigid registration and automatic segmentation and contouring could be important tools if a new planning is necessary during the radiation therapy of the same patient [5]. Registering old and new planning studies, and based on old contours delineated by a specialist, a new set of contours could be calculated using Origami.

The aim of this study is to present a new method for quantitative evaluation able to work with both convex and concave volumes without a tessellation step. Moreover, the method is able to provide a new set of accurate contours in combination with a non-rigid registration algorithm.

\section{MATERIALS AND METHODS}

\section{A. Clinical data}

The images used in the realization of this study come from prostate cancer radiotherapy planning studies. Delineation of the organs was accomplished by a radiologist outlining the contour of the organ in each slice. This procedure results in a set of two-dimensional (2D) contours that defines a 3D volume. The analysis always involves intrapatient images.

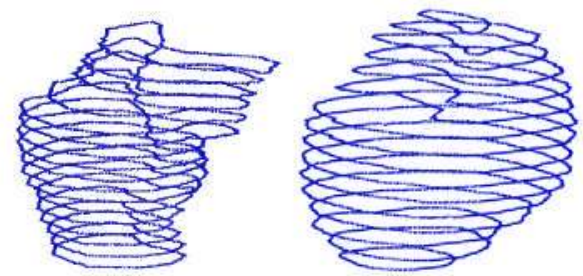

Fig. 13D volume of the prostate and seminal vesicles and bladder from $2 \mathrm{D}$ contours 
The method was used to evaluate prostate and bladder delineations in CT studies for radiotherapy patients. Organ surface and distance between volumes was scanned in spherical coordinates, using 64800 samples homogeneously distributed in the space, from the point of reference of the center of gravity of the organ in the image of reference.

\section{B. Error quantification}

Volume shape is quantified in terms of scalar samples of the 3D volume, as Remeijer et al. suggest. In this case, target volume is quantified by sampling the distance between the center of gravity and the surface of the organ for a large number of equidistant angles in the space. If the number of directions is sufficient, the distance between samples of the organ surface will be smaller than the contour point density, and a complete description of the organ will be provided.

Computing the center of gravity of each volume involves extracting the contours of interest from the planning study and creating a binary image. This way the center of gravity is computed along the whole volume involved by the surface of the organ, and not only by the surface.

The distance between two volumes in a single direction can be measured in several different ways, e.g. perpendicular to the organ surface from the intersection point, the shortest distance from the furthest intersection point, or simply the radial distance.

No one of these distances is shorter than the rest in all the cases, working with convex-concave volumes. Nevertheless, the really important task is to define a fixed and univocal distance that allows the reproducibility of the measure and the reconstruction of the scene, for what all the definitions of distance are useful, if the translated center of gravity of the organ is included in the reference volume. We have chosen radial distance since is the most intuitive one.

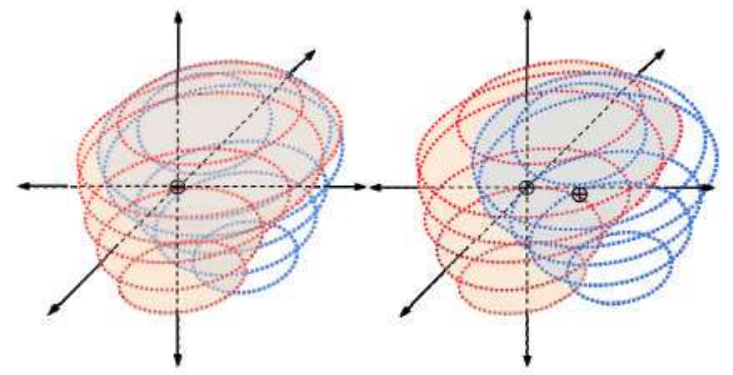

Fig. 2 a) Deformation measuring scheme, b) Distance measuring scheme.

Global displacement or simply deformation can be measured depending on the situation of the center of gravity of the translated organ: if the center of gravity is set on the center of reference, the deformation in any direction of the organ will be computed; if not, the global movement of the organ will be analyzed. Rotation and deformation of the organ cannot be separated in the analysis so rotation will be considered as a kind of deformation hereafter.

\section{Origami Method}

The proposed method [6] is based on the combination of two 2D analysis of the volume of the organ instead of one 3D analysis, measuring distances in each direction without a tessellation step that can introduce external errors. Origami method is composed by three steps:

- Axial contours: all contours drawn on axial slices are represented on a single plane and intersected by a set of polar rays centered on the projection of the point of reference on the plane. As a result, the frame of reference is homogeneously divided (Fig. 3a).
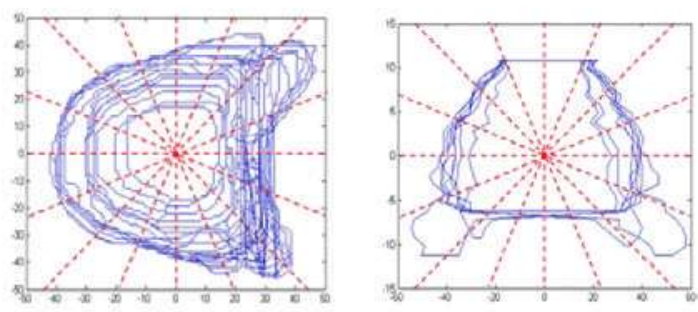

Fig. 3 a) Intersection of axial contours, b) Intersection of polar contours.

- Polar contours: the intersection points between each radial ray and every axial contour are raised to the height of each axial contour, and linked in order to form a new set of radial contours. The result is similar to intersecting the target volume with a set of radial planes of $\theta$-angle perpendicular to the axial slices (Fig. 4).

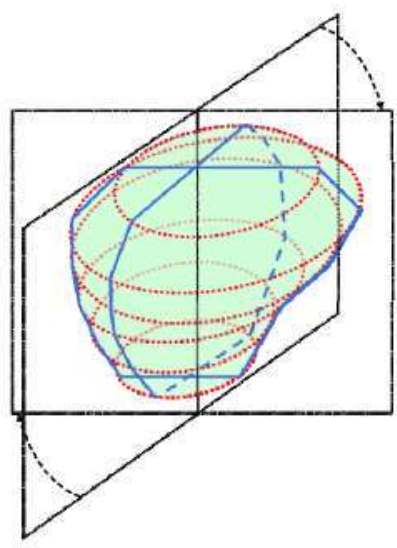

Fig. 43D representation of reconstruction of radial contours. 
- Surface sample computing: new radial contours are represented on a single plane folding down the Z-axis, and intersected by a new set of radial rays, again in a 2D frame of reference (Fig. 3b). The new set of radial rays has to take into account the different dimensions of the voxel in the tomographic images; usually the pixel dimensions in each slice are equal, but the distance between slices is higher. The volumetric coordinates of the control point are extracted from $\theta$-angle of the radial plane and $\varphi$-angle and rho ( $\rho$ ) from this last intersection.

\section{Results AND DICUSSION}

It is necessary a comprehensive method of data visualization because of the huge amount of simultaneous results. In this case two comprehensive $2 \mathrm{D}$ visualization methods have been used: bull's eye maps, used in nuclear medicine for the representation of the left ventricle, and polar maps, suggested in previous works.

Polar maps are used in order to represent the distance in each direction, assigning $\mathrm{x}$-axis to variable $\theta, \mathrm{y}$-axis to variable $\varphi$ and a color of the colorbar to variable $\rho$ in a cartesian representation, while bull's eye maps use a polar representation assigning P to variable $\varphi, \Theta$ to variable $\theta$ and a color of the colorbar to variable $\rho$.

Thus, the up side of the organ will be in $\varphi=90^{\circ}$ (resulting in the top of the polar map o the external ring of the bull's eye map), the down side in $\varphi=-90^{\circ}$ (equivalent to the bottom side of the polar map and the inner part of the bull's eye map), the front side of the organ will be in $\theta=0^{\circ}$ (center of the polar map and right of the bull's eye map) and the back side in $\theta= \pm 180^{\circ}$ (left and right sides of the polar maps and left of the bull's eye maps).

Polar maps and bull's eye maps are very suitable for the display of results for near spherical structures, but an inherent drawback of these representation methods is that only one value per direction can be represented. So, only the maximum value has been chosen to be represented in each direction.

The representation of an organ (Fig. 5) is the basis of the method to reconstruct the shape of the deformed organ or the global displacement (translation of center of gravity and deformation) of the organ between studies. This polar map represents the distance of each surface sample to the selected origin (center of gravity of the organ) for each direction, so data are always positive.

Volumetric distance between the prostate in a reference study and the prostate in the registered study is shown in Fig. 6. Distance is measured from the organ of reference, so data results can be positive or negative. Distance in each direction can be debt to a translation, a rotation or a deformation (or a combination of them) of the organ. It is impossible to know the global transformation suffered by the organ using only the data of this polar map, but combining it with $3 \mathrm{D}$ analysis of the organ of reference, it is possible to reconstruct the new position and shape of the organ, that is reconstruct the whole $3 \mathrm{D}$ scenery. This methodology cannot be used in case the displacement is so large that the center of reference is outside the displaced organ, but these translations are not common.

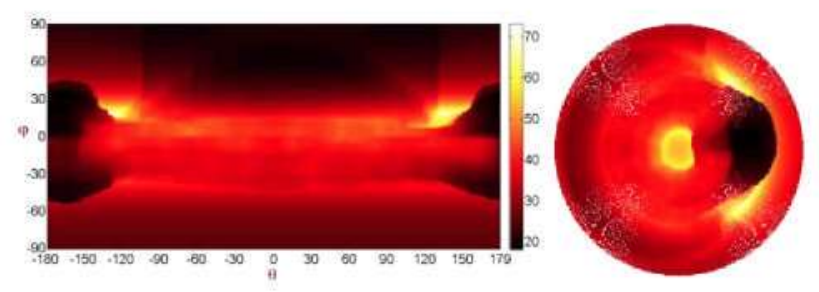

Fig. 5 3D analysis of prostate and seminal vesicles.

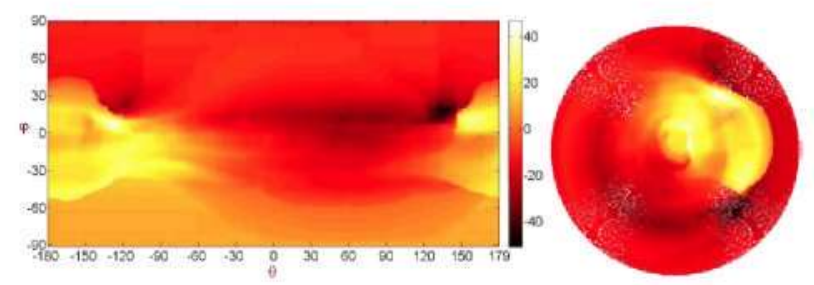

Fig. 6. Distance between the reference prostate and a displaced prostate.

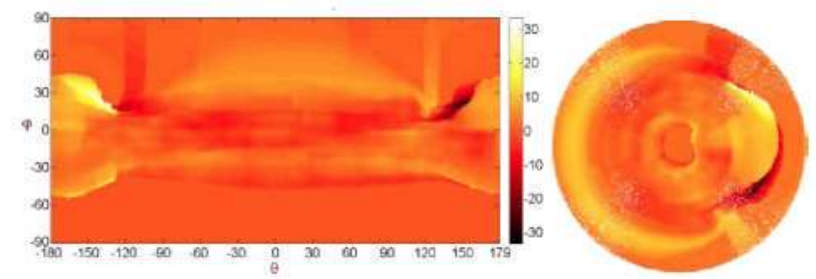

Fig. 7. Deformation and/or rotation of prostate and seminal vesicles.

When the center of gravity of the reference organ and the center of gravity of the displaced organ are set in the center of reference, only the deformation of the organ (Fig. 7) is computed. This measure can be very important in combination with Image Guided Radiation Therapy (IGRT) techniques that allow measuring the translation of the organ of interest between sessions.

As an example of the improvements in the organ reconstruction and analysis introduced by Origami method respect tessellation models, differences, in millimeters, between Convex Hull method with Delaunay triangulation and Origami method are shown as a polar map in Fig. 8. 

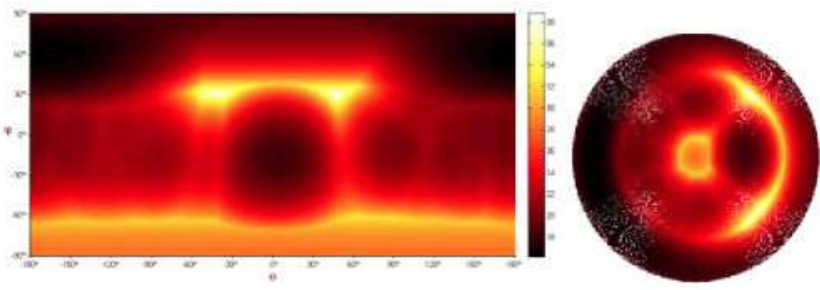

Fig. 8 3D analysis using Convex Hull

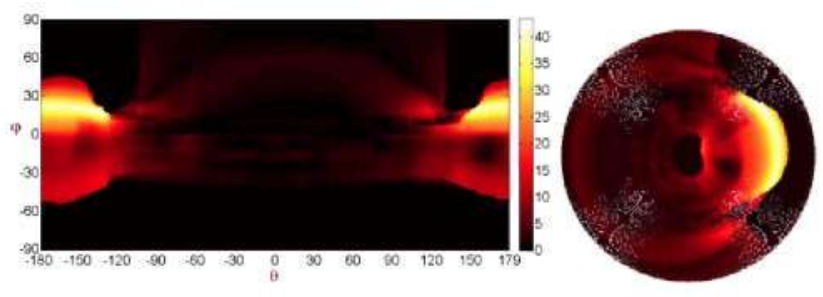

Fig. 9 Differences between Origami method and Delaunay triangulation.

The distance differences tend to be null in convex regions of the volume, especially in the top and down slices that are just planes. However, differences between both methods in the concave regions are significant, with the maximum around the seminal vesicles in the prostate. The concavity in the posterior side of the prostate, due to the deformation induced by the rectum, presents differences around $15 \mathrm{~mm}$, while concavities close to seminal vesicles show differences of more than $40 \mathrm{~mm}$ (lightest regions on the polar map). These are significant errors in a small organ such the prostate.

A $3 \mathrm{D}$ visualization method is suggested in order to overcome the drawback of the $2 \mathrm{D}$ visualization methods, where the registration error is represented as a color in a point of the reference organ, representing the distance between both volumes in that point.

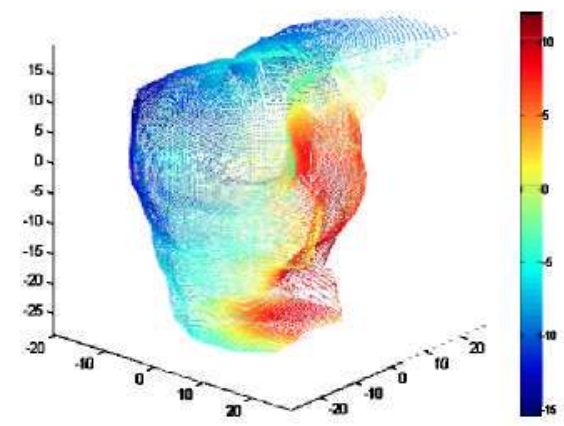

Fig. $103 \mathrm{D}$ visualization of the registration error

The results obtained by our method are 64800 control points per volume (one each solid angle of one square degree), in images of radiotherapy planning for prostate cancer, in less than one second using a PC with a Pentium 4 $3 \mathrm{GHz} \mathrm{CPU}, 1 \mathrm{GHz}$ RAM and a Matlab implementation.

\section{Conclusions}

Origami method has shown to be useful in the analysis of variations on target volume delineations, giving a full 3D description of the contours variability. The method works correctly in both convex and convex-concave structures, obtaining a better accuracy than other tessellation methods, with an exceptional time performance.

A univocal and intuitive definition of distance between convex-concave volumes has been set and tested, being useful to reconstruct the scene and/or deformation of the organ, and very intuitive visualization methods has been suggested for the representation of the distribution of the error.

\section{AckNOWLEDGMENT}

The authors want to thank Dr. Zapatero Laborda from the University Hospital of La Princesa, Madrid (Spain), for her collaboration.

\section{REFERENCES}

1. Zapatero A, Rios P, Minués R, Garcia-Vicente F. Dose escalation with three-dimensional conformal radiotherapy. (2006) Is more dose really better in high-risk patients treated with androgen deprivation? Clin Oncol (R Coll Radiol), Vol. 18, No 8, pp 600-607.

2. International Commission on Radiation Units and Mesasurements (ICRU). (1993) Report No 50. Prescribing, Recording and Reporting Photom Beam Therapy.

3. Remeijer P, Rasch C, Lebesque JV, van Herk M. (1999) A general methodology for three-dimensional analysis in target volume delineation. Medical Physics, Vol. 26, No 6, pp 331-340.

4. Pevsner A, Davis B et al. (2006) Evaluation of a deformable image matching method for quantifying lung motion in respirationcorrelated CT images. Medical Physics, Vol. 33, No 2, pp 369-376.

5. Lu W, Olivera G, et al. (2006) Deformable registration of the planning image (KVCT) and the daily images (MVCT) for adaptive radiation therapy. Phys. Med. Biol. Vol. 51, pp 4357-4374.

6. Rodriguez-Vila B, Gaya F, del Pozo F, Garcia-Vicente F, Gomez EJ. (2008) A new geometrical method for 3D evaluation of non-rigid algorithms for radiotherapy in prostate cancer. Computed Assisted Radiology and Surgery (CARS) 2008. Barcelona.

$\begin{array}{ll}\text { Author: } & \text { Francisco Gayá Moreno. } \\ \text { Institute: } & \text { E.T.S.I.T. - Polytechnic University of Madrid. } \\ \text { Street: } & \text { Ciudad Universitaria s/n. } \\ \text { City: } & \text { Madrid. } \\ \text { Country: } & \text { Spain. } \\ \text { Email: } & \text { fgaya@gbt.tfo.upm.es }\end{array}$

